\title{
EL SUJETO \\ HUMANO EN LA \\ ORGANIZACIÓN
}

\section{RESUMEN}

Este artículo pone al sujeto humano en el centro de la discusión del Comportamiento Humano Organizacional, mediante la búsqueda del factor común de los autores representativos en el tema, que revele cuál ha sido su preocupación predominante. Desde la tradición burocrática weberiana, la teoría organizacional ha sido testigo del objetivo lucrativo que se les ha dado a las organizaciones. Para los supuestos actuales, las formas legítimas de dominación estarían fundamentadas por la incapacidad de la mayoría de resolver los asuntos cotidianos, esto es, la consecución de rentabilidad. Se empieza a evidenciar que las organizaciones, lejos de ser un conjunto de acciones racionales, son lugares de decisiones poco racionales, más frecuentemente usadas para objetivos personales que comunitarios. La conclusión pone de presente un nuevo tema de discusión para la teoría organizacional: la concepción de un actor libre dentro de la organización.

Palabras clave: Sujeto; Poder; Dominación; Agente libre. 
MARCELA PÉREZ PAZMIÑO*

\section{THE HUMAN SUBJECT IN THE ORGANIZATION}

\section{ABSTRACT}

This article puts the human subject into the center of the discussion, by finding common factors of representative authors in the field, revealing what was their predominant concern. From the Weberian bureaucratic tradition, organizational theory has witnessed that economic target was in the very center of organizations. For current assumptions, legitimate forms of domination would be substantiated by the inability to resolve most everyday affairs, those that handle to the rentability. It starts to show that organizations, far from being a rational set of actions, are places of unsound decisions, most often used for personal than community goals. The conclusion brings a new topic of discussion for organizational theory: the concept of a free agent within the organization.

Keywords: Subject; Power; Domination; Free Agent.

\section{INTRODUCCIÓN}

Las organizaciones que originalmente fueron concebidas para la consecución de fines comunes (Crozier \& Friedberg, 1990) han sido usadas como elementos de dominación (Weber, 1960). Sin embargo, lo más presente en la sociedad actual es la organización; no podemos concebir nuestra actividad actual sino a través de organizaciones. Foucault (1988) ha asegurado que las organizaciones objetivan al sujeto humano al dividirlo según los intereses de los grupos de poder (Ceballos, 2000). Algunos autores han tomado en cuenta esta contradicción (Arendt, 2006; Foucault, 1988; Ramos, 2001) poniendo el acento de sus estudios en la liberación del sujeto humano en las organizaciones.

Este artículo pone en el centro de la discusión del Comportamiento Humano Organizacional precisamente al sujeto humano, mediante la búsqueda del factor común de autores representativos en el tema, a través de una revisión bibliográfica en el trabajo de Weber, Foucault, Crozier y Friedberg, que revele cuál ha sido su preocupación predominante.

El sujeto humano organizacional tiene, para este trabajo, las siguientes dimensiones: 1) es un sujeto reflexivo "entendido como aquel sujeto que afecta al objeto en su relación con este en tanto interviene de manera activa en el conocimiento y en la organización de los objetos", según una de las categorías de Jesús Ibáñez (Izquierdo Uribe, 1999, p. 242), y 2) siguiendo a Foucault, es un "individuo continuamente construido por las relaciones sociales, discursos y prácticas, es decir, construido por las relaciones conocimientos-poder" (Davel; Constant Vergara, 2001, p. 47). Esto permite posicionar al sujeto humano organizacional en
* Doctora (c), docente de la Universidad de Las Américas uDLA, Quito. Docente a tiempo parcial en la Universidad Andina Simón Bolívar, Quito. Ecuador. Correo-e: mm.perez@udlanet.ec

Recibido: 17 de marzo de 2015, aceptado: 19 de junio de 2015.

Para citar el artículo: Pérez, M. (2015). "El sujeto humano en la organización", en Sotavento MBA, n. ${ }^{\circ} 26$, pp. 26-32. DOI: http://dx.doi.org/10.18601/01233734. n26.03 
una perspectiva posmodernista (Chan, 2000), que se aleja del racionalismo con el que han sido construidas las teorías comportamentales de la organización.

\section{FUNDAMENTO TEÓRICO}

Este trabajo realiza una revisión bibliográfica en la producción de cuatro autores principales: Max Weber en su obra "Los tipos de dominación", escrita en 1960; Michel Foucault con "El sujeto y el poder", de 1988, y Michel Crozier y Erhard Friedberg en su obra conjunta "El actor y el sistema", producida en los años 80. Lo que se intenta es establecer un análisis comparativo entre los tres autores, acerca de su visión del sujeto humano en las organizaciones. Los tres autores han sido seleccionados por encontrar que sus posturas son coincidentes, y sus respuestas al problema del sujeto pueden dar nuevas luces a la teoría organizacional y en específico, al comportamiento organizacional.

\section{RESULTADOS Y DISCUSIÓN}

Desde la tradición burocrática weberiana, la teoría organizacional ha sido testigo del objetivo lucrativo que se les ha dado a las organizaciones. Weber (1960) señala que toda relación de dominación ocurre por dos motivos: arreglos racionales con relación a fines y arreglos racionales con relación a valores. El lucro es concebido como fin, pero justificado a través de intereses valorativos, que no pueden abandonarse por estar íntimamente ligados con la condición humana.

La dominación requiere ser legitimada por quienes hacen uso de ella y por quienes son sujetos de ella. Weber (1960) asegura que en la dominación burocrática, que atiende a roles, funciones y cargos, los empleados la legitiman cumpliendo con lo acordado: mientras más evidente es el tipo de dominación, menos conscientes son de ellas los dominados.

Merton (1960) señala que "la elección de los funcionarios superiores en una burocracia se hace por circunstancias políticas, y tiene por objeto influir en los fines de la organización, pero los medios técnicos para la consecución de los fines quedan en manos del personal burocrático permanente" (1960, p. 202). Merton asegura que esta cualidad de permanente es la causa de las ineficiencias de la burocracia, puesto que elimina la necesidad de competencia y mejoramiento. Así, las reglas se asumen sin mayor reflexión y las tareas se realizan irracionalmente.

Siguiendo la misma línea, Blau (1960) afirma que la cohesión social que se logra en medioambientes en los que predomina la igualdad básica de estatus, enfrenta a los retos que trae consigo la cooperación. Esta cohesión puede causar una suerte de parálisis consentida y asumida por los burócratas.

La visión racional encuentra la contradicción entre sujeción y subjetividad, que se debate entre la dominación y el control, y un sujeto humano que es un objeto de uso para la organización, pero que la define con su accionar.

Cuando se busca la organización ideal, se hace referencia a las preferencias personales, con lo que resulta una imagen que representa nuestro ego (Schwartz, 1987). Mientras más idealizada tengamos a la organización, más difícil es encontrar esa imagen, pudiendo ocasionar en el individuo depresión y aislamiento. El totalitarismo en la organización hace uso de este recurso idealizador, para crear ilusiones que se asemejen a la imagen del autor. Si bien se puede mostrar que el totalitarismo puede lograr eficiencia en ciertos momentos, no es menos cierto que también sumerge a los individuos en desequilibrios personales.

Las consecuencias de la dominación racional, según lo expresa Weber, se evidencian en que hay una separación entre fines y medios: los fines los establecen los dominadores, los medios 
están en las manos de los dominados; además, se evidencia una pérdida de competitividad, puesto que en niveles iguales de estatus no hay necesidad de competencia; esto ocasiona incremento del control sobre los dominados.

Foucault (1988) considera que ante toda dominación aparece una resistencia, que es una lucha por liberarse de la sumisión. Actualmente, dice Foucault, esta es la lucha más sobresaliente, porque la forma de dominación imperante es aquella relacionada con el poder político, que somete al individuo en función del bienestar de la colectividad. Si bien se refiere específicamente al Estado, reconoce que otras instituciones, como la Iglesia, fueron las originarias de este poder, que denomina "poder pastoral".

La intención del poder pastoral es la "salvación" del individuo a través de su adhesión a la comunidad, que puede ser tan grande como la nación o tan pequeña como la familia. A partir de aquí, podemos imaginar que el poder pastoral se ejerce en el ambiente organizacional, convirtiendo al sujeto humano en una extensión de la gran maquinaria productiva, que solo consigue su objetivo a través del sacrificio de la individualidad.

Deleuze (1999), evocando a Foucault, descubre que esta forma de proceder trata de recuperar la perdida competitividad, instituyendo entre los individuos una rivalidad interminable, a modo de sana competición, como una motivación excelente que contrapone unos individuos a otros y atraviesa a cada uno de ellos, dividiéndolo interiormente. La burocracia ahora ha sido remplazada por otros medios de dominación: "Ahora, el instrumento de control social es el marketing, y en él se forma la raza descarada de nuestros dueños. El control se ejerce a corto plazo y mediante una rotación rápida, aunque también de forma continua e ilimitada [...] El hombre ya no está encerrado sino endeudado" (Deleuze, 1999, p. 8).

La dominación política hace que el sujeto organizacional se pierda porque lo divide. Sin embargo, Foucault advierte, al igual que lo hiciera en su momento Weber, que la dominación acarrea ineficiencias y deseo de liberación.

Si se mira a la organización como una gran mesa de juego, tal como proponen Crozier y Fierdberg (1990), se podría entender que el individuo estaría en capacidad de administrar su propio juego, puesto que controla sus habilidades y, con ellas, las incertidumbres que se pudieran originar como parte de la estrategia y de las negociaciones propias de esta actividad. En ello está presente siempre el poder.

El juego del poder dentro de la organización se establece entre individuos (los jugadores), que interpretan su rol de acuerdo con sus intereses y recursos (Crozier \& Friedberg, 1990). Esto acarrea una serie de consecuencias que pueden preverse desde el punto de vista de Foucault: por un lado, el jugador es pieza clave del juego, no puede liberarse de esta circunstancia para participar; es decir, vuelve a ser parte de la comunidad practicante. Por otro lado, los recursos están limitados por la misma normativa que encierra las posibilidades de actuación libre de los jugadores.

El sujeto depende enteramente de las circunstancias; es manejado como un títere al arbitrio del dueño de la cancha de juego. Sin embargo, asegura Crozier, siempre hay un grado de libertad al que puede aferrarse este jugador menos aventajado. Hay un margen de incertidumbre, que este controla, el cual es inaccesible para los demás. Mientras más pertinente sea para el juego, más libertad tendrá de hacer uso de ese recurso.

Crozier y Friedberg (1990) señalan que el fundamento de la acción organizada es el poder: "Toda estructura de acción colectiva, por estar sostenida sobre las incertidumbres "naturales" de los problemas por resolver, se constituye como sistema de poder" (Crozier \& Friedberg, 1990, p. 2).

El poder y todas sus facetas, incluso las vergonzosas, son parte de la acción colectiva 
porque el campo de acción de la organización tiene una estructura social en la cual se tratan los problemas (Crozier \& Friedberg, 1990). Por ello, los autores sugieren mirar estas estructuras para indagar qué mecanismos operan para la resolución de los problemas.

En cierta forma, decir que no hay acción social sin poder no es más que otra manera de decir que no hay, y no puede haber, campo que no esté estructurado, pues si toda estructura supone, crea y reproduce poder, es decir desigualdades, relaciones de dependencia y mecanismos de control social, se puede afirmar además que tampoco puede haber poder sin estructuras, dado que estas proporcionan las protecciones necesarias para enfrentar los fenómenos de poder, es decir, para hacer posible la cooperación (Crozier \& Friedberg, 1990).

Esto significaría que los actores que operan en una organización estarían protegidos por su estructura, al mismo tiempo que limitados por ella (Bourdieu, 1996). Esta limitación es también una posibilidad de alcanzar objetivos comunes, puesto que lo que hacen los actores es aprovechar oportunidades que perciben en función de su capacidad. Entonces, la posibilidad de ejercer el poder depende de las estructuras y las reglas, las que originan zonas de incertidumbre, sobre las cuales se crean relaciones de poder.

Esta forma funcionalista de concebir a la organización ha dado lugar a las teorías que analizan a la organización a través de dividirla en departamentos, funciones, cargos, que ayudan a explicar, pero no a entender, la compleja realidad organizacional (Benson, 1977). Clegg (1987) concluye que una mirada inocua al poder, sin entender su lenguaje, de dónde proviene y a dónde va, nos lleva a caer en el mismo reduccionismo que se trata de evitar.

Bourdieu (1996) ha dejado sentado que las formas que toma la organización en el papel no son las mismas que toma en la realidad. Las personas dentro de espacios comunes tienden a ocupar sitios de acuerdo al cúmulo de capital que poseen. Aquellas que están en la posición más alta son las que han acumulado mayor capital económico, luego se distribuyen de acuerdo con la cantidad total de capital: económico, cultural y simbólico. Estas formas, lejos de ser reales, son interpretadas desde el subjetivismo bajo el propio "sentido de lugar" (sense of one's place), que no es más que la propia subjetividad ocultando la realidad.

Un intento por desterrar la mirada de dominación del poder dentro de la organización es propuesta por Knights y Roberts (1982), sugiriendo que en las relaciones que se establecen dentro y fuera de la organización se manifiesta el poder que es inherente a la capacidad de acción de cada uno. Los autores generan una propuesta de mirar al poder como un intercambio dentro de las relaciones, que se puede alcanzar mediante negociación. El acuerdo es contingente, esto es, dependiendo de la capacidad y recursos de cada parte, los arreglos varían en el tiempo. Esto, dicen los autores, garantizaría una posibilidad de mirarse cada uno como contribuyente al quehacer organizacional.

La racionalidad instrumental opera en sentido inverso al que se pretende conseguir, porque se da por supuesto que los actores van a representar adecuadamente sus papeles. Pero no todos en la maquinaria conocen su guion o no están dispuestos a seguirlo, por no convenir a sus intereses, afectando el desempeño esperado. Entonces, se valoran y se justifican los mecanismos de control, convertidos ahora en herramientas inclusive automatizadas, para conseguir un mínimo de consenso necesario para la producción organizacional.

Se empieza a evidenciar que las organizaciones, lejos de ser un conjunto de acciones racionales, son lugares de decisiones poco racionales, más frecuentemente usadas para objetivos personales que comunitarios. Es así que el sujeto, que no deja de serlo en ningún momento, es concebido como pieza, más o menos importante, del gran complejo organizativo 
que, contradictoriamente, habría sido construido para su "salvación". Crozier insiste en que la organización mirada como juego, con sus reglas, estrategias y herramientas, permite descubrir en los jugadores aquellas incertidumbres que le otorgan algún grado de libertad. Esta forma de establecer las estrategias y las acciones del juego organizativo, permite entender la acción organizada, que no es más que la búsqueda de un común denominador dentro de la organización, o el encuentro de la razón de existir de la organización.

Una versión diferente de la organización conformada por algo más que estructuras, objetivos y dominación se hace necesaria para comprender por qué siguen vigentes. Esta nueva perspectiva deberá incluir las múltiples y diversas relaciones que se establecen entre los actores, sin permitirse perder de vista que estos son individuos, que se juntan por motivos tan diversos como sus propias singularidades.

Los diferentes autores revisados coinciden en su punto de vista pesaroso respecto del accionar actual de las organizaciones. Sobre todo la preeminencia del poder como potestad de unos pocos, la dominación basada en la sujeción, el control como mecanismo de presión y el ocultamiento de los conflictos.

Los autores trasladan el pensamiento de la organización hacia la necesidad de crear ambientes que faciliten la cooperación, el trabajo conjunto y la búsqueda del sentido propio dentro de la organización. Dejar de lado las prácticas soterradas, que no solo no ayudan, sino que entorpecen la creación de espacios comunitarios para el cumplimiento de objetivos, es una prioridad.

Se hace evidente la necesidad de mirar el comportamiento humano en la organización a través de otros sistemas de referencia, que den la posibilidad de liberación del sujeto humano, que confíen en su versatilidad para hacerse cargo de situaciones complejas, en fin, que lo hagan parte de la organización, como fuera en el origen.

\section{REFERENCIAS}

Arendt, H. (2006). Sobre la violencia (Primera re.). Madrid: Alianza Editorial S.A.

Benson, J. (1977). "Organizations: A Dialectical View", en Administrative Science Quarterly, 22(1), 1-21. Retrieved from http://www.jstor.org/ stable/10.2307/2391741

Blau, P. M. (1960). "La dinámica de la estructura burocrática", en FLACSO (Ed.), Sociologia del poder. Santiago de Chile: Editorial Andrés Bello.

Bourdieu, P. (1996). Cosas dichas (Primera re.). Barcelona, España: Gedisa.

Chan, A. (2000). "Redirecting Critique in Postmodern Organization Studies: The Perspective of Foucault", en Organization Studies, 21(6), 1059-1075.

Clegg, S. (1987). "The Language of Power and the Power of Language", en Organization Studies, 8(1), 61-70.

Crozier, M. \& Friedberg, E. (1990). El actor y el sistema: las restricciones de la acción colectiva (pp. 1-15). Alianza Política.

Davel, E. y Constant Vergara, S. (2001). Gestao com pessoas e subjetividade (p. 257). Sao Paulo: Editora Atlas S.A.

Deleuze, G. (1999). Post-scriptum sobre las sociedades de control. In P. Editions de Minuit (Ed.), Conversaciones (pp. 1-9). Valencia:T. G. Ripoll S.A.

Foucault, M. (1988). "El sujeto y el poder", en Revista Mexicana de Sociología, 50(3), 3-20.

Izquierdo Uribe, A. (1999). "Espacio-temporalidad y ominijetividad: una aproximación espitemológica", en Nómadas, (11), 241-248.

Knights, D. \& Roberts, J. (1982). "The Power of Organization or the Organization of Power?", en Organization Studies, 3(1), 47-63.

Merton, R. K. (1960). "Estructura burocrática y personalidad", en FLACSO (Ed.), Sociologia del Poder. Santiago de Chile: Editorial Andrés Bello.

Ramos, A. G. (2001). Modelos de homem e teoria administrativa. PUC-PR/Mestrado em Administração/série monográfica: "Caderno de Ciências Sociais Aplicadas", (3), 3-12. 
Schwartz, H. (1987). "On the Psychodynamics of Organizational Totalitarianism", en Journal of Management, 13(1), 41-54.

Shrivastava, P., Mitroff, I. \& Alvesson, M. (1987). "Nonrationality in Organizational Actions", en International Studies of Management ..., XVII(3),
90-109. Recuperado el 20 de septiembre de 2014 http://www.jstor.org/stable/10.2307/40397071

Weber, M. (1960). "Los tipos de dominación", en FLACSo (Ed.), Sociologia del Poder (Antologías.). Santiago de Chile: Editorial Andrés Bello. 